\title{
La conception benjaminienne de la réécriture chez Juan Mayorga
}

Erwan Burel

\section{(2) OpenEdition}

1 Journals

Édition électronique

URL : https://journals.openedition.org/cher/2049

DOI : 10.4000/cher.2049

ISSN : 2803-5992

Éditeur

Presses universitaires de Strasbourg

\section{Édition imprimée}

Date de publication : 29 mai 2018

Pagination : $43-50$

ISBN : 979-10-344-0019-5

ISSN : 1968-035X

\section{Référence électronique}

Erwan Burel, «La conception benjaminienne de la réécriture chez Juan Mayorga », reCHERches [En ligne], 20 | 2018, mis en ligne le 01 décembre 2021, consulté le 02 décembre 2021. URL : http:// journals.openedition.org/cher/2049; DOI : https://doi.org/10.4000/cher.2049

\section{(c) (i) (2)(2)}

Ce(tte) œuvre est mise à disposition selon les termes de la Licence Creative Commons Attribution Pas d'Utilisation Commerciale - Partage dans les Mêmes Conditions 4.0 International. 


\section{La conception benjaminienne de la réécriture chez Juan Mayorga}

ERWAN BuREL ${ }^{1}$

$\mathrm{D}$ ans son texte "Conservación y creación. Respuesta diferida a un actor chino $»^{2}$ (2016: 145-151), Juan Mayorga se penche sur le problème de la réécriture et, pour ce faire, n'hésite pas à s'appuyer sur les réflexions concernant le langage (et notamment la traduction) de Walter Benjamin. Mais avant d'en arriver à ces considérations benjaminiennes, il nous rappelle l'anecdote qui l'a amené à penser le concept de réécriture.

En 2000, lors d'un voyage en Chine, il assiste aux répétitions de la Compagnie Nationale de l'Opéra de Beijing et rencontre, à l'issue de la représentation, l'acteur protagoniste. Ce dernier lui raconte que son père était déjà un acteur, et que son grand-père, lui aussi, était un acteur, et que son arrière-grand-père, lui aussi, en était un, et ainsi de suite. Depuis des décennies, tous ces fils d'acteurs ne cessent de jouer le même rôle à l'Opéra, dans les mêmes costumes, dans la même mise en scène, avec les mêmes musiques. Depuis des décennies, se répète sur les planches de l'Opéra la même représentation de la même œuvre. Et c'est justement ce qui interpelle notre dramaturge espagnol: contrairement à ce qu'on a l'habitude de faire en Occident, ici, en Chine, les textes classiques ne sont pas adaptés, il n’y a pas d'actualisation des pièces du passé. En ce sens, Mayorga affirme:

Il est fort probable que l'acteur chinois m'ait oublié. Cependant, il est devenu pour moi un véritable problème. Je pense souvent à lui. Je pense à lui chaque fois que je travaille à l'adaptation d'un texte classique, et chaque fois que je me propose d'écrire une nouvelle pièce. L'acteur chinois, et son père, et son grand-père, et son arrière-grand-père, font irruption et me demandent: Pourquoi? Pourquoi adapter les textes classiques, qui sont précisément des classiques parce qu'ils sont

1 Professeur agrégé d'espagnol, docteur en Études espagnoles et spécialiste du théâtre de Juan Mayorga, Université de Strasbourg.

2 «Conservation et création. Réponse différée à un acteur chinois». 
capables d'entrer en résonance avec n'importe quelle époque? Et pourquoi écrire un mot de plus si les grandes œuvres du passé renferment déjà tout ce qui vaut la peine d'être dit? (Mayorga 2016: 146) ${ }^{3}$

Mayorga répond à ces problématiques en faisant appel à la notion de traduction. En effet, il estime que celui qui s'attèle à l'adaptation d'un texte classique est un traducteur: celui qui adapte un texte du passé fait vivre ce texte dans un temps différent de celui où il a été écrit initialement. D’une manière générale, tous ceux qui s'engagent dans la transmission problématique d'une pièce du passé, nous dit le dramaturge (2016: 147) - et il entend par "pièce» tout objet de la culture (œuvre théâtrale, picturale, musicale, etc.) - peut être considéré comme un traducteur. Pour lui, la traduction est un déplacement, une opération à la fois conservatrice et créatrice, qui a lieu non seulement dans le travail d'adaptation, mais également dans le processus même de création:

Le mouvement propre à la traduction, qui comprend autant la continuité (la transmission) que l'interruption (la rupture), exclut l'éternel retour, la répétition infinie, mais aussi la prétention de parler un langage adamique, primitif. À la limite - dans la mesure où non seulement on traduit des mots, mais aussi des idées, des formes, des gestes... - chaque création peut être considérée comme une traduction. Car dans chaque œuvre, d'autres, qui l'ont précédée, sont sauvées [...]. Finalement, l'histoire de la culture peut être vue comme une incessante migration d'un pays à un autre, d'une époque à une autre, d'un art à un autre: comme une traduction infinie. (Mayorga 2016: 147-148)

La référence à un langage adamique, primitif, ainsi que l'idée de rédemption des œuvres du passé dans les œuvres actuelles (voire futures), ne sont pas étonnantes ici lorsque l'on comprend que Juan Mayorga appuie sa réflexion sur la philosophie du langage de Walter Benjamin, et notamment sur sa conception de la traduction que le dramaturge explicite comme suit:

Pour Walter Benjamin, le plus important pour le traducteur est ce qui est impossible à traduire. Car, lorsque la langue d'arrivée s'avère insuffisante, le souci de fidélité à la langue de départ demande au traducteur de faire travailler la langue d'arrivée, de l'ouvrir. Dans ces moments-là, fidélité et liberté coïncident. La meilleure traduction entre deux langues serait, selon Benjamin, celle capable de faire que ces deux langues se reconnaissent comme fragments d'un langage supérieur. En ce sens, dans la traduction est représenté un langage pur, la langue de la vérité, la fin même de la philosophie. Chaque traduction est pour Benjamin non seulement une forme de survie de l'œuvre originale, mais également la représentation du mouvement infini des langues vers une langue vraie. L'espoir de ce langage absolument autre réside donc entre les lignes, dans l'espace de ce qui n'est encore écrit dans aucune des langues. (Mayorga 2016: 148)

Afin d'affiner ces réflexions sur la traduction chez Walter Benjamin, et d'éclairer ainsi l'idée de réécriture chez Juan Mayorga, étudions en détail la question du langage chez le philosophe judéo-allemand.

3 Toutes les citations de ce texte de Juan Mayorga dans le corps de notre travail sont traduites en français par nos soins. 


\section{La question du langage chez Walter Benjamin}

Ce qu'il faut tout d'abord bien avoir à l'esprit, c'est que la philosophie benjaminienne du langage repose sur une interprétation du récit biblique de la Genèse. Dans Sur le langage en général et sur le langage humain (2000a : 142-165), Benjamin cherche à montrer que toute la réalité créée est d'ordre linguistique.

Selon le philosophe, il existe trois strates de réalité qui nivellent l'être des choses. Il y a d'abord le niveau de Dieu, cet être créateur, divin et omniscient, où tout le langage consiste en une nomination créatrice (le Verbe divin). Ce langage nomme les choses et, en les nommant, les crée et les connaît immédiatement. Ce monde des choses, nommé et créé par Dieu, est le monde de la nature et constitue la deuxième strate de réalité. Cependant, ce monde de la nature, «où les choses adviennent à l'être comme langage [...] [puisqu'elles] sortent tout droit du langage divin qui leur donne être» (Tackels 2009: 678), est un monde muet, un monde qui a perdu tout pouvoir de nomination/création. La troisième strate de réalité correspond à celle du premier homme. Dieu créa le monde de la nature et son langage silencieux, puis Adam. Contrairement au monde muet de la nature, Adam fut doté du pouvoir de nomination et, dans la continuité de la création divine, nomma effectivement les choses ou, plus précisément, prononça leur nom:

Dieu transmet à Adam ce fabuleux pouvoir d'entendre dans le mutisme de la nature l'écho du Nom divin qui présida à sa création. À l'écoute de la nature, Adam reçut de Yahvé le pouvoir de (re)nommer les choses, de proférer leur nom, ce nom par lequel Dieu lui-même les créa. (Tackels 2009: 678-679)

Ce pouvoir de nomination hérité de Dieu n'a plus de fonction créatrice. Il s'agit pour Adam de se réapproprier les noms divins, de les connaître. La nomination acquiert ici une fonction de connaissance. De plus, en prononçant le Nom, "l'homme adamique communique à Dieu la part communicable de l'essence spirituelle des choses: il traduit le langage muet, anonyme des choses dans son langage articulé ${ }^{\star}$ (2009: 679). Mais en prononçant le Nom, l'homme adamique communique aussi à Dieu

la part communicable de sa propre essence spirituelle, qui n'est autre que cette puissance de traduction, ce pouvoir de donner nom au sans-nom et sonorité au sans-son. L'essence spirituelle de l'homme, qu'il donne à voir dans son langage, coïncide très précisément avec son langage même, avec son essence linguistique. [...] En suivant cette logique, le langage n'apparaît plus du tout comme un moyen de communication, un instrument censé dénoter, signifier quelque chose qui lui serait extérieur [...]. Ce qu'il communique, ce n'est jamais que le caractère éminemment langagier des choses elles-mêmes et le caractère intrinsèquement «nommable» de l'homme, en un mot le fait que tout son être est dirigé vers et par cette nominabilité, en tant que pouvoir de donner nom. (Tackels 2009: 679)

4 Dans toutes les citations de l'ouvrage de Bruno Tackels (2009), les mots en italiques ont été mis sous cette forme par l'auteur. 
Suivant le déroulement de la Genèse, Benjamin sépare les hommes de l'homme adamique grâce à l'épisode de la chute, du péché originel, par lequel on entre dans l'ère de la (fausse) connaissance du bien et du mal, du jugement. Concernant le langage, il identifie cette chute dans la tour de Babel et la dispersion des langues, où l'on passe du langage de Noms au langage de mots. Ce langage n'est plus synonyme de connaissance mais de jugement et de divisions. Contrairement au nom, écho du Nom divin donné aux choses par lequel l'homme adamique connaissait immédiatement le monde de la nature, le mot, lui, n'est plus immédiat à la chose car il en devient un signe. Le mot devient un instrument par lequel l'homme tente désespérément de saisir la chose, sans jamais réussir. Autrement dit, le mot et la chose, la linguistique et l'ontologique, sont définitivement séparés, et les langues post-babéliennes - le langage humain - tendent indéfiniment, sans jamais l'atteindre, vers le pur langage adamique.

Benjamin aperçoit dans la pratique de la critique, ainsi que dans celle de la traduction, une manière de s'approcher de ce langage pur, où la dimension transmissive ou communicationnelle de la parole tend à disparaître.

Il développe sa pensée autour de la notion de critique dans sa thèse doctorale Le concept de critique esthétique dans le romantisme allemand (Benjamin 2008), et trouve chez les romantiques une définition de la critique pouvant servir sa propre conception du langage. Pour les romantiques, la critique d'une ouvre n'est pas le jugement porté sur elle mais la méthode d'accomplissement de l'œuvre elle-même (Mayorga 2003: 30). Cette idée suppose la survie de l'œuvre par rapport à son auteur car, en effet, il s'agit non pas d'évaluer l'œuvre à partir d'un patron particulier mais bien de repérer en elle son germe critique, la réflexion qui lui est immanente et qui, de par les lectures successives qui sont faites de l'œuvre tout au long de l'histoire, s'amplifie dans un processus infini et tend vers toujours plus de vérité et de beauté (idem). La critique romantique est, en quelque sorte, une complémentation et un parachèvement de l'œuvre qui, indéfiniment, tend vers l'absolu de l'art, et donc, pour Benjamin, vers un langage pur et adamique.

En ce qui concerne le phénomène de la traduction, il l'évoque dans son essai sur le langage lorsqu'il se réfère à l'homme adamique et à son langage de noms, traduction du langage muet de la nature. Il remarque l'objectivité de cette traduction, dans le sens où il existe une continuité entre ces langages, celui de la nature et celui de l'homme adamique, puisqu'ils proviennent tous les deux de la parole divine créatrice (Mayorga 2003: 33). Cependant, concernant les langues humaines, ces langues post-babéliennes survenues après la chute, cette continuité n'existe plus. Dès lors, l'essence de la traduction ne peut se restreindre au problème de la reproduction du sens (idem). Dans son essai La tâche $d u$ traducteur (2000b: 244-262), Benjamin nous montre que c'est paradoxalement par le geste de traduction, par le contact entre les langues, que doit être brisé le carcan du sens pour que l'homme puisse entrevoir ce qu'était le langage avant la chute: 
[...] l'idée de Benjamin est simple: le sens, loin de révéler la vérité, l'opacifie et l'enfouit au plus profond de lui-même. La fonction de la traduction sera donc de casser la gangue du sens et de la signification, pour rapatrier le langage dans l'espace de vérité. L'objectif du traducteur tient dans cette formule paradoxale: c'est pour lutter contre le sens qu'existe et doit se développer le geste de traduction. Ou plus précisément, le poids du sens de chaque langue doit se purifier et s'alléger au contact de la langue étrangère, en tant que langue d'accueil. Le sens partiel qui vise "quelque chose» disparaît (comme poids) au moment où la traduction le met en contact avec la langue de l'autre, avec le sens partiel de l'autre langue. $\mathrm{Ce}$ "quelque chose» visé par toute langue finie (c'est-à-dire visant le sens absolu, sans jamais l'atteindre comme tel, dans sa totalité de sens), ce "quelque chose» visé partiellement par toutes les langues humaines n'est autre que le pur langage adamique. (Tackels 2009: 689-690)

La tâche de la traduction serait donc de faire apparaître ce pur langage de noms mais, évidemment, il s'agit là d'un idéal, jamais atteint, à l'instar des langues elles-mêmes qui, on l'aura compris, visent toutes - mais ne l'atteignent jamais - ce pur langage:

Le traducteur véritable, au fil de l'histoire des langues, œuvre pour la survie de ce langage étouffé [le langage adamique], pour sa délivrance, pour son apparition comme pur visé, c'est-à-dire non visé par un visant, par le caractère visant, instrumental des langues finies. [...] En accédant à la langue de l'autre, l'original trouve un écho, non de lui-même, mais de ce dont lui-même est l'écho: le pur visé, l'essence ultime du langage, le nom. (Tackels 2009: 690)

Fort de ces développements benjaminiens sur le langage, nous pouvons nous demander ce qu'il en est de la réécriture chez Juan Mayorga.

\section{La réécriture chez Juan Mayorga}

Dans les réflexions benjaminiennes sur le langage, il y a en creux une dimension temporelle, caractéristique d'ailleurs de la pensée benjaminienne en général, et qui a à voir avec l'idée de rédemption messianique. Cette teneur messianique, utopique, voire révolutionnaire, est patente dans les fameuses Thèses sur le concept d'histoire (Benjamin 2000c: 427-443), mais inonde toute l'œuvre benjaminienne ou, du moins, illumine de l'intérieur tous ses écrits. Le temps auquel aspire Benjamin est un temps ouvert, non linéaire, où puisse apparaître, en un éclair, la figure du Messie, et où l'humanité serait sauvée. Pour le philosophe, la traduction - tout comme la critique d'ailleurs - serait, selon lui, un vecteur d'apparition de la vérité, elle permettrait de

faire mûrir la sur-vie des œuvres de l'histoire, c'est-à-dire de faire émerger la vie souterraine qui anime sourdement le devenir mortifère des œuvres de langage. Pour les donner à voir sous un jour salvateur. [...] Aux êtres de langage, il ne reste que la possibilité de raviver ce qui est en mémoire de ce moment initial [où le langage n'était que pure révélation] - ranimer une perception originelle de ce qui a toujours disparu. (Tackels 2009: 691-692) 
Le langage des hommes est un langage déchu. C'est un langage qui a perdu tout pouvoir de nomination et donc de connaissance immédiate du monde. Cependant, c'est dans ce langage même, dans cette déchéance dont témoigne le langage humain, que peut être révélée à l'homme son histoire, son appartenance passée à un monde de vérité et de beauté, et, par conséquent, c'est dans la déchéance même du langage humain qu'est possible le salut de l'humanité:

Par un geste profondément révolutionnaire, tourné vers l'avenir, le mot - la totalité des mots - peut atteindre la noblesse du nom. En ranimant le passé gardé en mémoire par le mot, le voyageur - qui devient par le fait même historien - atteste que dans le mot travaille révolutionnairement le nom. Le langage, fût-il déchu, et grâce à cette déchéance même, sauve le passé véritable et rend possible la rédemption du monde. La théorie benjaminienne du langage reprend l'idée qui traverse tous les textes de jeunesse: la "déchéance» (le "déclin» d'une civilisation - qui se marque dans son rapport au langage) est immanquablement chargée d'une force rédemptrice. Il ne faut pas entendre par là que l'attention au langage nous délivrerait de la chute, mais plutôt qu'à même la matérialité du monde déchu se délivre, se dispense l'éclat d'un langage bienheureux, la fulgurance d'un sens linguistique logé dans l'histoire. (Tackels 2009: 688)

Réécrire, traduire, faire la critique des œuvres du passé, tout cela consiste finalement à travailler à l'apparition (utopique) d'un temps salvateur où serait possible - pour parler encore une fois en termes benjaminiens - la grande citation entre le présent et le passé. C'est d'ailleurs une des conclusions à laquelle arrive Juan Mayorga dans son texte sur la réécriture que nous avons commenté en introduction:

Traduire: au lieu de partir en chasse dans la réserve luxuriante de l'Histoire pour exhiber ensuite les captures comme un butin de guerre, construire une citation périlleuse entre deux temps. Une citation dont le résultat ne serait pas l'absorption d'un temps par un autre, mais un troisième temps, aussi imprévisible que l'est le passé. Car, en effet, le passé est imprévisible. Le passé est ouvert. (Mayorga 2016: 149)

La réécriture, telle que nous la comprenons à la lumière des développements benjaminiens sur le langage et - tout cela est lié - sur l'histoire (celle des hommes et de la culture), est omniprésente dans le travail de Juan Mayorga. Chez notre dramaturge, elle concerne d'abord les pièces qui, effectivement, sont des reprises, des adaptations, des versions ou des compilations de textes préexistants, de grands mythes de la littérature dramatique, de classiques. Nous pouvons classer ces œuvres en deux catégories:

- Les «versions» ou adaptations (où Juan Mayorga n'est mentionné que comme simple adaptateur puisqu'il ne travaille que légèrement le texte initial) : Hécuba (Euripide); La vida es sueño et El monstruo de los jardines (Calderón de la Barca); Fuente Ovejuna et La dama Boba (Lope de Vega); El Rey Lear (Shakespeare); Natán el Sabio (Lessing); Woyzeck (Büchner); El Gran Inquisidor (Dostö̈evski); El enemigo del pueblo (Ibsen); Platonov 
(Tchekhov); Divinas palabras (Valle-Inclán); Ante la Ley (Kafka); La visita de la vieja dama (Dürrenmatt).

- Les «à partir de» (où Juan Mayorga est cette fois-ci mentionné en tant qu'auteur puisqu'il met en place des procédés de réécriture plus poussés, mais où la parenté originale de l'œuvre est toujours forte et explicite): El sonámbulo (a partir de Sobre los ángeles de Rafael Alberti); Wstawać (Homenaje a Primo Levi); Job (a partir del Libro de Job y de textos de Elie Wiesel, Zvi Kolitz y Etti Hillesum); Primera noticia de la catástrofe (a partir de Bartolomé de las Casas).

Cela concerne ensuite les pièces dites "originales", où le phénomène de réécriture est plus profond - lorsqu'il repose sur une transformation assumée du texte initial, comme dans Fedra ou Palabra de perro - ou bien plus subtil lorsqu'il fait partie intégrante de l'univers dramatique de la pièce et n'apparaît plus seulement comme un procédé de création littéraire auquel l'auteur a recours mais également comme une composante de la fiction théâtrale. Concernant ce dernier cas, nous pouvons penser à des pièces comme La lengua en pedazos, El chico de la última fila, Cartas de amor a Stalin, El traductor de Blumemberg et El crítico, qui font reposer les phénomènes de réécriture sur des mises en abyme du langage ou sur des intrigues construites autour de l'idée même d'écriture, de traduction ou de critique. Nous pourrions aussi évoquer des pièces comme Himmelweg, El cartógrafo et El jardín quemado, qui abordent la question de la réécriture par le biais de la mise en scène de l'écriture de l'histoire et de la construction de la mémoire historique ${ }^{5}$.

Le spectre de la réécriture chez Mayorga est donc très large et passe par une multitude de procédés tous plus différents les uns que les autres, selon qu'il cherche à adapter un texte classique à la scène contemporaine, à créer une pièce à partir de matériaux littéraires préexistants ou à problématiser dans ses œuvres le langage et l'écriture. Mais la réécriture concerne, en dernier ressort, la pratique même de l'écrivain puisque Mayorga ne cesse de réécrire ses pièces au fil du temps. Cette réécriture est motivée le plus souvent par les différentes mises en scène réalisées par lui-même (La lengua en pedazos, Reikiavik, El cartógrafo) ou par autrui et a comme objectif d'améliorer le passage du texte à la scène. Cette pratique traduit surtout chez notre auteur une attention permanente au langage et renvoie, tel que nous l'avons expliqué précédemment, à une conception benjaminienne du temps, qui veut que le temps réécrive les œuvres du passé ou, pour le dire autrement, qui veut que le temps ouvre le passé et fasse surgir de nouvelles significations. Plus que tout autre art, le théâtre a cette capacité

5 Le but de cet article est d'apporter un éclairage théorique en étudiant la conception benjaminienne de la réécriture chez Juan Mayorga. Nous pourrions approfondir les différents aspects cités pour ces pièces "originales" mais cela nous demanderait de procéder à des analyses dramaturgiques, ce qui n'est pas le lieu ici. La plupart des pièces mentionnées peuvent être consultées dans l'ouvrage Teatro 1989-2014 (Mayorga 2014). 
de dialectiser le passé avec le présent, et Mayorga en est bien conscient. Son œuvre est empreinte d'un souci - artistique et philosophique - du temps, et le phénomène de réécriture y trouve une place particulièrement intéressante.

\section{Bibliographie}

Benjamin W., [1916] 2000a, Sur le langage en général et sur le langage humain, Euvres I, traduit par De Gandillac M., Rusch P., Rochlitz R., Paris, Gallimard. Benjamin W., [1923] 2000b, La tâche du traducteur, Euvres I, traduit par De Gandillac M., Rusch P., Rochlitz R., Paris, Gallimard.

Benjamin W., [1940] 2000c, Thèses sur le concept d'histoire, Euvres III, traduit par De Gandillac M., Rusch P., Rochlitz R., Paris, Gallimard.

Benjamin W., [1920] 2008, Le Concept de critique esthétique dans le romantisme allemand, Paris, Flammarion.

Mayorga J., 2003, Revolución conservadora y conservación revolucionaria. Política $y$ memoria en Walter Benjamin, Barcelona, Anthropos.

Mayorga J., 2014, Teatro 1989-2014, Segovia, La uÑa RoTa.

Mayorga J., 2016, Elipses, Segovia, La uÑa RoTa.

Tackels B., 2009, Walter Benjamin. Une vie dans les textes, Arles, Actes Sud. 